\title{
INFLUÊNCIA DE TRATAMENTO TÉRMICO E SISTEMAS DE EMBALAGENS NA QUALIDADE DE LICHIA 'BENGAL''
}

\author{
VALÉRIA DELGADO DE ALMEIDA ANJOS², \\ SILVIA REGINA DE TOLEDO VALENTINI ${ }^{3}$, ELIANE APARECIDA BENATO ${ }^{4}$
}

RESUMO - Estudaram-se oito tratamentos quanto à eficiência de banhos hidrotérmicos com ou sem adição de produtos químicos na conservação da qualidade de lichias acondicionadas em bandejas de PET com tampa (BT) e cobertas com filme de PVC de $15 \mu \mathrm{m}(\mathrm{BF})$, sendo submetidas a banhos hidrotérmicos, tais como:T1-BT e T2-BF - sem tratamento; T3-BT- água a $55^{\circ} \mathrm{C}$ por $20 \mathrm{~s}$; T4-BF- água a $55^{\circ} \mathrm{C}$ por $20 \mathrm{~s}$; T5$\mathrm{BT}$ - água a $55^{\circ} \mathrm{C}$ por $10 \mathrm{~s}$ e em ácido cítrico $60 \%$ por $10 \mathrm{~s}$; T6-BF- água a $55^{\circ} \mathrm{C}$ por $10 \mathrm{~s} \mathrm{e} \mathrm{banho} \mathrm{de} \mathrm{ácido}$ cítrico $60 \%$ por $10 \mathrm{~s}$; T7-BT- ácido cítrico $60 \%$ por $20 \mathrm{~s}$; T8-BF-ácido cítrico $60 \%$ por $20 \mathrm{~s}$. Avaliaram-se as principais alterações físicas, químicas, sensoriais e presença de doença em lichias armazenadas a $4^{\circ} \mathrm{C}$ e a $25^{\circ} \mathrm{C}$. De forma geral, ao final do estudo, as lichias ficaram ligeiramente menos ácidas, com leve aumento no $\mathrm{pH}$ e com discreta redução de sólidos solúveis, e após dois dias a $25^{\circ} \mathrm{C}$ todos os tratamentos apresentaram infestação por fungos.Os tratamentos T5, T6, T7 e T8 foram considerados inaceitáveis $(\geq 4)$ a partir de 15 dias; T1, T2, T3 e T4 aceitáveis até 21 dias, pela avaliação visual. Quanto à manutenção da cor vermelha, os melhores tratamentos foram T1 e T4. O tratamento T2 apresentou os maiores valores de firmeza da casca, mostrando que o filme de PVC protegeu contra a perda de firmeza, de umidade e ao murchamento. A menor perda de massa foi nos tratamentos T6 e T8 $(0,4 \%)$, ambos com filme de PVC, e a maior perda de massa foi nas amostras T5 (5,6\%) acondicionadas nas bandejas perfuradas, além de apresentar menor firmeza na casca. Termos para a indexação: Litchi chinensis Sonn, pós-colheita e escurecimento.

\section{INFLUENCE OF HEAT TREATMENT SYSTEMS AND PACKAGING IN THE QUALITY OF LYCHEE 'BENGAL'}

\begin{abstract}
Litchi fruits cv. Bengal were submitted to hydrothermal treatments and packed in PET trays with lid (BT) and covered with PVC film $15 \mu \mathrm{m}(\mathrm{BF})$. The treatments were : T1-BT- and T2-BF without hydrothermal treatment; T3-BT-water at $55^{\circ} \mathrm{C}$ for $20 \mathrm{~s}$, T4-BF-water at $55^{\circ} \mathrm{C}$ for $20 \mathrm{~s}$, T5-BT-water at $55^{\circ}$ $\mathrm{C}$ for $10 \mathrm{~s}$ plus citric acid $60 \%$ for $10 \mathrm{~s}$, T6-BF-water at $55^{\circ} \mathrm{C}$ for $10 \mathrm{~s}$ plus citric acid $60 \%$ for $10 \mathrm{~s}$, T7-BTcitric acid $60 \%$ for $20 \mathrm{~s}$ and T8-BF- citric acid $60 \%$ for 20 s. Changes in the main physical, chemical and sensory characteristics and the presence of disease in litchi stored at $4{ }^{\circ} \mathrm{C}$ and $25^{\circ} \mathrm{C}$ were evaluated. At the end of the study, litchi fruits were slightly less acid, with a slight increase in $\mathrm{pH}$ and a slight reduction of the soluble solids content. After two days at $25^{\circ} \mathrm{C}$ all fruits were infected by molds. The treatments T5, T6, T7, T8 were considered unacceptable (score $\geq 4$ ) from the $15^{\text {th }}$ day, T1, T2, T3 and T4 were acceptable up to 21 days by visual assessment. The treatments $\mathrm{T} 1$ and $\mathrm{T} 4$ resulted in the best red color. Treatment T2 had the highest values of firmness of the pericarp, showing that the PVC film protected against loss of firmness, moisture and wilting. The weight loss was lower for the treatments T6 and T8 (0.4\%) both with PVC film, and more than T5 (5.6\%) with PET tray with lid.
\end{abstract}

Index terms: Litchi chinensis, postharvest and browning.

\footnotetext{
${ }^{1}$ (Trabalho 307-13). Recebido em : 26-08-2013. Aceito para publicação em: 12-08-2014.

${ }^{2}$ Pesquisadora Científica do Instituto Agronômico de Campinas de Tecnologia de Alimentos. E-mail: vanjos@ital.sp.gov.br ${ }^{3}$ Pesquisadora Científica do Instituto Agronômico de Campinas de Tecnologia de Alimentos. E-mail: valentini@iac.sp.gov.br ${ }^{4}$ Pesquisadora Científica do Instituto Agronômico de Campinas de Tecnologia de Alimentos. E-mail: ebenato@biologico.sp.gov.br
} 


\section{INTRODUÇÃO}

O fruto da lichieira (Litchi chinensis Soon.), a lichia, é originário da China e tem alto valor comercial nos mercados nacional e internacional (JIANG et al., 2004). De característica subtropical a tropical, a lichia começou a ser produzida comercialmente no Brasil em 1970, em São Paulo. O maior produtor de lichia no Brasil é o Estado de São Paulo, com produção também nos Estados de Minas Gerais, Paraná e Bahia, com um total de área plantada em 2004 de 1.000 ha (MOTTA, 2009). O volume total comercializado de lichia na Ceasa de São Paulo, levantado pela SEDES - Seção de Economia e desenvolvimento da CEAGESP, cresceu 181 toneladas em 2002 para 974 toneladas em 2012 - quase cinco vezes. $\mathrm{O}$ ano de maior produção foi 2009, com 2.595 toneladas - um volume 13 vezes superior ao de 2000 (CEAGESP-HORTIBRASIL, 2014). A safra é entre novembro e janeiro, alternando com a safra no exterior, que ocorre nos meses de maio a agosto. Sendo assim, o Brasil poderá, em futuro próximo, abastecer o mercado externo com lichias na entressafra (EMBRAPA, 2009).O fruto em questão apresenta tamanho e formato variáveis que podem ser esféricos, de cônico ao cordiformes, com fino, duro e indeiscente pericarpo desenhado com saliências. Quando maduro, o fruto apresenta externamente cor vermelho-brilhante e textura rugosa. A porção comestível é de coloração branca creme translúcida, muito suculenta e aparência gelatinosa, aromática, sendo caracterizada pelo sabor agridoce (HOLCROFT; MITCHAM; 1996); (TODA FRUTA, 2005). As variedades mais plantadas no Brasil são as chinesas, a saber: Bengal, Americana e Brewster. A variedade Bengal é originada e selecionada na Flórida a partir da variedade indiana Purbi. No Brasil, a colheita dá-se entre a metade de novembro e princípio de janeiro. O fruto, que é cordiforme, tem peso médio de $21 \mathrm{~g}$, coloração vermelho-brilhante, textura macia, doce e moderadamente suculenta. A porcentagem de polpa é cerca de $60 \%$ do fruto, com semente grande (CAVALLARI, 2009).

A lichia é rica em minerais e vitaminas, e contém a cada $100 \mathrm{~g}$ de polpa os seguintes teores: $82,0 \mathrm{~g}$ de água, 65 calorias, $0,8 \mathrm{~g}$ de proteína, $0,4 \mathrm{~g}$ de gordura, Ca (10 mg), P (29 mg), Fe (0,3 mg), Na (3 $\mathrm{mg}), \mathrm{K}$ (170 mg), Tiamina (0,5 mg), Riboflavina (0,6 $\mathrm{mg})$, Niacina (0,6 mg) e Vitamina C (95 mg), açúcar (10 a 12\%) (EMBRAPA, 2009; MOTTA, 2009). Sendo a lichia não climatérica, os frutos devem ser colhidos no ponto ideal de consumo quando estes apresentam coloração vermelho-brilhante devido ao pigmento antocianina presente na casca da fruta
(ZHANG et al., 2000).

Após a colheita, inicia-se o processo de escurecimento da casca de 2 a 3 dias, e após a colheita, o epicarpo torna-se desidratado, apresentando coloração marrom e consequente fissuras no epicarpo (HUAG, et al., 2005). De acordo com Zang et al. (2000), é difícil estocar a fruta por tempo maior do que 3 a 4 dias na temperatura ambiente, sem tratamento apropriado. O principal problema póscolheita da lichia é o supracitado escurecimento do pericarpo. Embora seja somente em aspecto de aparência, torna os frutos impróprios para a venda no mercado da UE e dos EUA (HOLCROFT; MITCHAM, 1996).

Esse escurecimento tem sido atribuído à degradação da antocianina, devido à ação das enzimas oxidativas, polifenoloxidase (PPO), peroxidase (POD) e ácido ascórbico oxidase (JIANG et al., 2004; HOLCROFT; MITCHAM, 1996; ZHANG et al., 2000). Vários tratamentos têm sido sugeridos visando à redução do escurecimento em lichia e controle de infestação fúngica, tais como: imersão em água quente; uso de ácidos ascórbico, cítrico, tartárico, oxálico, com mistura de glutadiona, quitosanas; uso de poliaminas; fungicida; lecitina; ceras; compostos à base de enxofre e acondicionamento em embalagens com atmosfera modificada; imersão em ácido clorídrico e a fumigação com produtos à base de enxofre, sendo estes últimos evitados devido aos efeitos colaterais à saúde do consumidor (HOLCROFT; MITCHAM, 1996; JIANG; CHEN, 1994; SIVAKUMAR; KORTEN, 2006; LICHER et al., 1999; DUCAMP-COLLIN et al., 2008).

Dentro deste contexto, o objetivo deste estudo é avaliar a eficiência de banhos hidrotérmicos, com ou sem adição de produtos químicos, na conservação da qualidade de lichias armazenadas a $4^{\circ} \mathrm{C}$ e a $25^{\circ} \mathrm{C}$, durante 21 dias, para a primeira temperatura e 2 dias para a segunda, seguindo esta ordem.

\section{MATERIAL E MÉTODOS}

Lichias cv. Bengal, cultivadas no município de Santa Branca-SP, foram enviadas para o Laboratório de Pós-Colheita do ITAL, em Campinas-SP, onde foram submetidas a oito tratamentos, sem ou com banhos hidrotérmicos, utilizando bandejas de PET com tampa perfurada (BT) e bandejas de PET cobertas com filme de PVC de $15 \mathrm{~mm}$ (BF) com aproximadamente $500 \mathrm{~g}$, conforme descrito a seguir: T1-BT e T2-BF - lichias sem tratamento; T3-BTágua a $55^{\circ} \mathrm{C}$ por $20 \mathrm{~s}$; T4-BF- água a $55^{\circ} \mathrm{C}$ por 20 $\mathrm{s}$; T5-BT- água a $55^{\circ} \mathrm{C}$ por $10 \mathrm{~s}$ e banho em ácido cítrico $60 \%$ em água por $10 \mathrm{~s}$; T6-BF- água a $55^{\circ} \mathrm{C}$ 
por $10 \mathrm{~s}$ e banho de ácido cítrico $60 \%$ em água por $10 \mathrm{~s}$; T7-BT- ácido cítrico $60 \%$ em água por $20 \mathrm{~s}$; T8BF-ácido cítrico $60 \%$ em água por $20 \mathrm{~s}$. Após cada tratamento, as lichias foram submetidas à eliminação da água superficial por meio de ventilação forçada. As amostras de cada tratamento foram armazenadas a $4,0 \pm 1,0^{\circ} \mathrm{C}$ e $86,5 \pm 1,6 \%$ UR por 21 dias e por mais dois dias a $25 \pm 2,0^{\circ} \mathrm{C}$ e $80 \pm 5 \%$ UR. Estas foram avaliadas quanto à aparência, defeitos superficiais, mudança de coloração vermelha para marrom, manchas ou presença de doença, utilizando uma escala de 1 a 5, com os respectivos valores: 1- sem defeitos, vermelho intenso; 2 - ligeiramente marrom até $10 \%$; 3 - cor marrom em até $25 \%$ da superfície; 4 - cor marrom e defeitos entre 25 e $50 \%$ da superfície (limite da aceitabilidade); 5 - cor marrom e defeitos em mais que $50 \%$ da superfície (CARO; JOAS, 2005). Para o diagnóstico de doença e isolamento e identificação do patógeno, apenas separaramse as amostras infectadas (ALFENAS; MAFIA, 2007). A cor externa foi medida diretamente no fruto em colorímetro Minolta CR300, pelo sistema CIELab (configuração $\mathrm{d} / 0$, iluminante D65, ângulo de observação de $2^{\circ}$ ), obtendo-se os valores de $L^{*}$ (luminosidade), $+\mathrm{a}^{*}$ (vermelho) e $+\mathrm{b}^{*}$ (amarelo). A manutenção da cor vermelha da lichia foi o principal foco deste estudo, uma vez que é por este atributo que o consumidor expressa a intenção da compra, associando a cor à qualidade da fruta (FERREIRA, 1991).

Para medir a firmeza da amostra, utilizouse do texturômetro TA-XT2i (Marca Stable Micro Systems), operando com software Texture Expert com modo de operação compressão/Return to start com leitura direta na casca das amostras, $6 \mathrm{~mm}$ de compressão, corpo de prova $\mathrm{P} 35$, submetidos à velocidades de pré-teste, teste e pós-teste de 2,0; 0,6 e $2,0 \mathrm{~mm} / \mathrm{s}$, respectivamente. O teor de sólidos solúveis ( ${ }^{\circ}$ Brix) foi determinado em refratômetro RF SENSOR SR 400 digital com compensação de temperatura para $20^{\circ} \mathrm{C}$. A acidez titulável em ácido málico foi determinada em $10 \mathrm{~g}$ de suco extraído dos frutos. $\mathrm{O} \mathrm{pH}$ foi determinado em pHmetro Micronal (INSTITUTO ADOLFO LUTZ, 2005). Determinou-se a perda de massa dos frutos a cada época, para cada tratamento, pelo método gravimétrico. O planejamento experimental consistiu em 8 tratamento, 7 épocas $(2 ; 5 ; 8 ; 12 ; 15 ; 18$ e 21 dias após a colheita), sendo 2 parcelas por época, 6 frutos para análise física e 10 frutos para avaliação visual e diagnóstico de incidência de doença. Os resultados para cada tratamento, em cada época, foram submetidos à análise estatística pelo programa SAS, pela Análise de Variância e teste de Tuckey, ao nível de 5\% de significância.

\section{RESULTADOS E DISCUSSÃO}

A Tabela 1 apresenta os resultados obtidos da avaliação visual de cada tratamento durante $o$ armazenamento. Os tratamentos T5-BT, T6-BF, T7BT e T8-BF foram considerados inaceitáveis $(\geq 4)$ a partir de 15 dias; T1- BT, T2-BF T3-BT até 21 dias, e T4-BF acima de 21 dias pela avaliação visual, confirmando a eficiência do tratamento hidrotérmico com ou sem ácido na prevenção da mudança de cor da lichia. As amostras tratadas com ácido cítrico T5, T6, T7 e T8 apresentaram-se com tonalidade vermelhorosada e alguns pontos marrons, mas de forma geral protegeu contra a alteração da cor do epicarpo para marrom-claro, o que normalmente ocorre na ausência de tratamento. Em lichias cv. Kwaï Mi tratadas com ácidos orgânicos, tais como ácido tartárico, cítrico com pH 0,8, 1,0 e 1,3, minimizaram-se o escurecimento enzimático e a perda de massa das frutas, o índice de 5 ( $>50 \%$ de escurecimento) foi atingido aos 15 dias com as lichias estocadas a $10^{\circ} \mathrm{C}$ (JOAS et al., 2005). A eficiência do tratamento com ácidos orgânicos, ácidos cítrico, tartárico combinados com quitosana foi eficiente na manutenção da cor vermelha de lichias (Lichi chinensis) cv. Kwaï Mi (CARO; JONAS, 2005).

Os resultados para os parâmetros de cor CIELab vermelha $\left(+a^{*}\right)$ e amarela $\left(+b^{*}\right)$ e luminosidade $\left(\mathrm{L}^{*}\right)$ são apresentados nas Tabelas 2,3 e 4 . Os tratamentos T1-BT (lichias sem tratamento) e $\mathrm{T} 4-\mathrm{BF}$ (banho com água a $55^{\circ} \mathrm{C}$ por $20 \mathrm{~s}$ ) apresentaram os maiores valores para a cor vermelha aos 21 dias de estocagem $(27,6)$, e os tratamentos T5,T6 e T8, os menores valores de cor vermelha $(22,5)$. Houve diferença estatística entre os tratamentos $\mathrm{T} 1 \mathrm{e} \mathrm{T} 4$ com os tratamentos $\mathrm{T} 6 \mathrm{e}$ T8 para a cor vermelha ao final do estudo, devido provavelmente ao tratamento com ácido que alterou a cor das lichias para coloração mais rosada, alterando o pigmento do epicarpo. Houve pouca alteração na cor amarela da lichia. O tratamento T4-BF (banho em água a $55^{\circ} \mathrm{C}$ por $20 \mathrm{~s}$ ) foi o que garantiu a manutenção da cor amarela até o final do estudo, apresentando o valor de 10,3 em comparação ao valor inicial de 10,4 . O menor valor final para a cor amarela foi de 8,1 para o tratamento T3-BT (água a $55^{\circ} \mathrm{C}$ por $20 \mathrm{~s}$ ). Os tratamentos, ao final do estudo, não apresentaram diferença significativa ao nível de $5 \%$ de significância quanto à cor amarela. A luminosidade $\left(\mathrm{L}^{*}\right)$ indica quanto a amostra muda de cor pelo escurecimento ou clareamento. A lichia, neste estudo, não apresentou variação importante neste parâmetro e não houve 
diferença estatística significativa ao final do estudo. A cor vermelha da lichia pode ser preservada por, no mínimo, 3 semanas, com o uso de formulação apropriada, o que permite inclusive o transporte marítimo substituindo tratamentos com compostos de enxofre (DUCAMP-COLLIN et al., 2008). Quanto aos tratamentos, Joas et al. (2005) mostraram que a cor vermelha e a luminosidade são favorecidas pelo uso do banho com ácido tartárico $\mathrm{pH} 0,8$. Os valores de firmeza da casca oscilaram durante a estocagem para todos os tratamentos. O valor inicial de firmeza da amostra foi de 30,3 N. Os tratamentos T1-BT, T2BF, T4-BF, T7-BT e T8-BF apresentaram os maiores valores na última época de estudo, respectivamente: $35,4 \mathrm{~N} ; 35,9 \mathrm{~N} ; 38,3 \mathrm{~N} ; 33,3 \mathrm{~N}$ e $34,7 \mathrm{~N}$, entretanto não houve diferenças estatísticas entre os tratamentos a $5 \%$ de significância ao final de 21 dias. $\mathrm{O}$ tratamento T3-BT apresentou menor valor de firmeza $(27,7 \mathrm{~N})$ e o tratamento T4-BF apresentou o maior valor de firmeza ( 38,3N) (Figura 1). Verifica-se que houve acentuada queda no valor de sólidos solúveis ao longo do estudo, comparando com o valor inicial de 19,4 ${ }^{\circ}$ Brix. Não houve diferença estatística entre os tratamentos ao final de 21 dias de estocagem em todos os tratamento, entretanto o maior valor, obtido foi para o tratamento T2-BF (lichias sem tratamento) $\left(17,8^{\circ} \mathrm{Brix}\right)$, e o menor valor para o tratamento T4-BF (água a $55^{\circ} \mathrm{C}$ por $\left.20 \mathrm{~s}\right)\left(17,3^{\circ} \mathrm{Brix}\right)$ (Figura 2). Segundo Chitarra e Chitarra (2005), o consumo de açúcares aumenta em decorrência das alterações fisiológicas durante o armazenamento, devido à respiração e transpiração das frutas após a colheita. Os valores médios da acidez titulável, ao longo do estudo, apresentaram inicialmente ligeiro aumento, partindo de 0,265 g/100 g em ácido málico e atingindo $0,387 \mathrm{~g} / 100 \mathrm{~g}$ em ácido málico, a oito dias de armazenamento, decaindo em seguida até 21 dias para 0,29 g/100 g em ácido málico. A variação dos valores médios de $\mathrm{pH}$ ao longo da estocagem foi de 4,29 inicialmente, com queda no oitavo dia de estocagem (4,07), atingindo no final o valor de 4,34, corroborando as observações do valor da acidez, uma vez que o pH e a acidez têm uma relação inversamente proporcional, ou seja:quanto mais alto o pH, a menor acidez (Figura 3).

A queda da acidez e o aumento de $\mathrm{pH}$ de lichias cv. Bengal foram observados por Silva et al. (2012), tratados com ácidos e cobertos com filmes naturais de fécula de mandioca e de PVC. O consumo de ácidos orgânicos são inerentes às perdas fisiológicas, tendo como consequência a redução da acidez e o aumento do $\mathrm{pH}$, porém podem ter pequeno aumento devido ao processo de maturação (CHITARRA;CHITARRA, 2005). A perda de massa apresentou a seguinte ordem crescente: $0,43 \%$ de perdas para T6-BF e T8-BF, $0,67 \%$ de perda de massa para T4-BF, $1,00 \%$ de perda de massa para T2-BF, 2,80\% de perda de massa para T7-BT, 4,6\% de perda de massa para T1-BT, 4,7\% de perda de massa para T3-BT e 5,6\% de perda de massa para T5-BT. Segundo Chitarra e Chitarra (2005), perdas de massa na ordem de 3 a 6 \% são suficientes para causar um marcante declínio na qualidade para comercialização, o que se observa nos tratamentos T7-BT, T1-BT, T3-BT e T5-BT, que foram os que mais apresentaram perdas no presente trabalho. Em trabalho com lichias cv. Kwai Mi, com a perda de massa, quando tratadas com ácido tartárico e cítrico em diferentes pHs $(0,8,1,0$ e 1,3) em combinação com a quitosana, observaram-se valores em torno de $7 \%$ durante oito dias de estocagem a $10^{\circ} \mathrm{C}$ (CARO; JOAS, 2005).

Em trabalho de Joas et al. (2005), uma boa correlação de $\mathrm{R}^{2}=0,8977$ - entre a perda de massa e o escurecimento do epicarpo é apresentada.Nesse estudo, os autores observaram 9,3\% de perda de massa após 13 dias a $10^{\circ} \mathrm{C}$ em lichias cv. Kwaï Mi não tratadas. De acordo com Joas et al. ( 2005), a perda de massa não deve exceder a $4 \%$, correspondente a $20 \%$ de perda de massa do epicarpo para permanecer comercialmente estável. No presente estudo, o uso das bandejas de PET cobertas com filme de PVC reduziu a perda de massa, enquanto as embalagens com tampa perfurada possibilitaram maior troca com ambiente de armazenamento, acarretando maior perda de massa dos frutos (Figura 4). Após a retirada das amostras da câmara de $4^{\circ} \mathrm{C}$, estas foram armazenadas a $25^{\circ} \mathrm{C}$ e $80 \%$ de UR, por 02 dias, apresentando, após este período, mudança intensa de coloração e com presença de fungos. Os microrganismos patogênicos isolados das podridões e identificados nos frutos foram: levedura, Rhizopus sp., Lasiodiplodia sp., Colletotrichum sp. e Cladosporium sp. 
TABELA 1 - Avaliação visual de lichias 'Bengal' submetidas a tratamento térmico e sistemas de embalagens e armazenadas a $4^{\circ} \mathrm{C}$, por 21 dias.

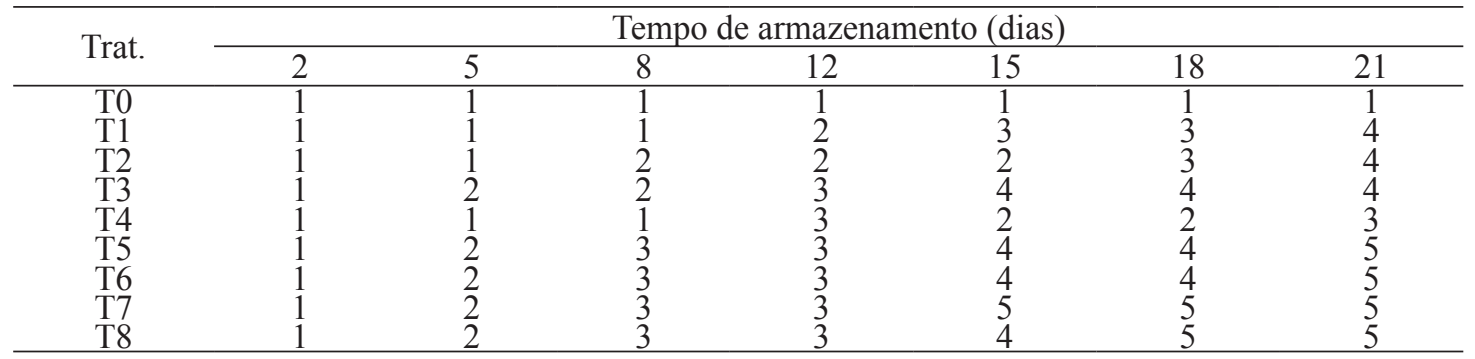

1-sem defeitos, vermelho intenso; 2 - ligeiramente marrom até 10\%; 3 - cor marrom em até 25\% da superfície; 4 - cor marrom e defeitos entre 25 e $50 \%$ da superfície (limite da aceitabilidade); 5 - cor marrom e defeitos em mais que $50 \%$ da superfície.

TABELA 2 - Valores médios/desvio-padrão de cor vermelha $\left(+\mathrm{a}^{*}\right)$ das lichias 'Bengal' submetidas a tratamento térmico e sistemas de embalagens e armazenadas a $4^{\circ} \mathrm{C}$ por 21 dias.

\begin{tabular}{cccccccc}
\hline \multirow{2}{*}{ Trat. } & \multicolumn{7}{c}{ Tempo de estocagem (dias) } \\
\cline { 2 - 7 } & 2 & 5 & 8 & 12 & 15 & 18 & 21 \\
\hline T1 & $27,1 / 1,4 \mathrm{~A}$ & $28,6 / 2,4 \mathrm{~A}$ & $27,8 / 1,6 \mathrm{AB}$ & $27,4 / 2,2 \mathrm{~A}$ & $29,1 / 2,5 \mathrm{~A}$ & $26,0 / 2,1 \mathrm{~A}$ & $27,6 / 1,3 \mathrm{~A}$ \\
T2 & $26,3 / 1,5 \mathrm{~A}$ & $29,3 / 1,4 \mathrm{~A}$ & $26,62 / 2,8 \mathrm{AB}$ & $28,2 / 3,0 \mathrm{~A}$ & $27,6 / 3,3 \mathrm{~A}$ & $26,3 / 3,7 \mathrm{~A}$ & $24,6 / 1,7 \mathrm{AB}$ \\
T3 & $26,6 / 2,0 \mathrm{~A}$ & $26,9 / 2,2 \mathrm{~A}$ & $24,3 / 2,6 \mathrm{~B}$ & $25,9 / 2,7 \mathrm{~A}$ & $29,0 / 1,6 \mathrm{~A}$ & $22,9 / 3,2 \mathrm{~A}$ & $23,7 / 2,5 \mathrm{AB}$ \\
T4 & $27,9 / 1,5 \mathrm{~A}$ & $25,9 / 1,5 \mathrm{~A}$ & $29,3 / 1,6 \mathrm{AB}$ & $27,7 / 3,8 \mathrm{~A}$ & $28,0 / 3,7 \mathrm{~A}$ & $25,6 / 2,5 \mathrm{~A}$ & $27,6 / 3,3 \mathrm{~A}$ \\
T5 & $27,8 / 2,2 \mathrm{~A}$ & $29,3 / 3,5 \mathrm{~A}$ & $26,9 / 2,4 \mathrm{AB}$ & $28,4 / 2,3 \mathrm{~A}$ & $27,4 / 2,6$ & $26,4 / 2,9 \mathrm{~A}$ & $22,5 / 3,9 \mathrm{AB}$ \\
T6 & $27,6 / 3,5 \mathrm{~A}$ & $28,7 / 2,2 \mathrm{~A}$ & $26,3 / 2,3 \mathrm{AB}$ & $24,0 / 1,5 \mathrm{~A}$ & $26,7 / 1,4 \mathrm{~A}$ & $22,6 / 2,4 \mathrm{~A}$ & $22,5 / 3,2 \mathrm{~B}$ \\
T7 & $28,3 / 2,1 \mathrm{~A}$ & $25,9 / 2,2 \mathrm{~A}$ & $25,8 / 3,0 \mathrm{AB}$ & $27,8 / 1,9 \mathrm{~A}$ & $26,2 / 2,4 \mathrm{~A}$ & $24,3 / 2,3 \mathrm{~A}$ & $23,6 / 3,3 \mathrm{AB}$ \\
T8 & $27,6 / 3,1 \mathrm{~A}$ & $26,8 / 2,8 \mathrm{~A}$ & $25,6 / 3,9 \mathrm{~A}$ & $25,1 / 1,4 \mathrm{~A}$ & $26,0 / 1,7 \mathrm{~A}$ & $23,2 / 3,9 \mathrm{~A}$ & $22,5 / 1,7 \mathrm{~B}$ \\
\hline
\end{tabular}

TABELA 3 - Valores médios/desvio- padrão de cor amarela $\left(+b^{*}\right)$ das lichias 'Bengal' submetidas a tratamento térmico e sistemas de embalagens e armazenadas a $4^{\circ} \mathrm{C}$ por 21 dias.

\begin{tabular}{cccccccc}
\hline & \multicolumn{7}{c}{ Tempo de estocagem (dias) } \\
\cline { 2 - 7 } Trat. & 2 & 5 & 8 & 12 & 15 & 18 & 21 \\
\hline T1 & $9,6 / 1,2 \mathrm{~A}$ & $10,2 / 1,0 \mathrm{~A}$ & $8,8 / 1,1 \mathrm{~B}$ & $10,9 / 2,1 \mathrm{~A}$ & $10,9 / 1,7 \mathrm{AB}$ & $9,4 / 1,2 \mathrm{AB}$ & $8,7 / 1,1 \mathrm{~A}$ \\
T2 & $8,1 / 1,0 \mathrm{~A}$ & $10,1 / 1,5 \mathrm{~A}$ & $10,6 / 1,8 \mathrm{AB}$ & $10,6 / 2,6 \mathrm{~A}$ & $11,0 / 1,5 \mathrm{AB}$ & $9,4 / 1,1 \mathrm{AB}$ & $8,5 / 1,6 \mathrm{AB}$ \\
T3 & $8,7 / 0,7 \mathrm{~A}$ & $9,4 / 1,3 \mathrm{~A}$ & $9,6 / 1,7 \mathrm{AB}$ & $9,2 / 1,4 \mathrm{~A}$ & $11,9 / 2,0 \mathrm{AB}$ & $8,5 / 1,4 \mathrm{AB}$ & $8,1 / 0,9 \mathrm{AB}$ \\
T4 & $9,4 / 1,0 \mathrm{~A}$ & $11,6 / 3,2 \mathrm{~A}$ & $10,8 / 2,1 \mathrm{AB}$ & $11,0 / 2,7 \mathrm{~A}$ & $9,8 / 2,8 \mathrm{~B}$ & $9,5 / 2,4 \mathrm{AB}$ & $10,3 / 1,5 \mathrm{~A}$ \\
T5 & $9,5 / 1,4 \mathrm{~A}$ & $10,3 / 1,9 \mathrm{~A}$ & $10,0 / 1,8 \mathrm{AB}$ & $9,8 / 1,4 \mathrm{~A}$ & $9,2 / 1,7 \mathrm{~B}$ & $10,3 / 1,6 \mathrm{BB}$ & $8,5 / 2,1 \mathrm{AB}$ \\
T6 & $9,0 / 1,4 \mathrm{~A}$ & $10,4 / 1,6 \mathrm{~A}$ & $11,1 / 0,7 \mathrm{AB}$ & $8,8 / 0,8 \mathrm{~A}$ & $11,1 / 0,9 \mathrm{AB}$ & $9,4 / 1,6 \mathrm{AB}$ & $8,3 / 3,1 \mathrm{~B}$ \\
T7 & $9,8 / 1,9 \mathrm{~A}$ & $8,9 / 1,5 \mathrm{~A}$ & $11,1 / 1,2 \mathrm{AB}$ & $10,3 / 0,8 \mathrm{~A}$ & $12,0 / 1,4 \mathrm{AB}$ & $8,7 / 1,0 \mathrm{AB}$ & $8,7 / 1,8 \mathrm{~A}$ \\
T8 & $9,6 / 1,0 \mathrm{~A}$ & $10,9 / 2,1 \mathrm{~A}$ & $12,0 / 1,5 \mathrm{~A}$ & $9,6 / 0,5 \mathrm{~A}$ & $12,9 / 0,9 \mathrm{~A}$ & $9,3 / 0,6 \mathrm{~A}$ & $9,0 / 2,0 \mathrm{~A}$ \\
\hline
\end{tabular}

Medias/desvio-padrão seguidas da mesma letra na vertical não apresentam diferença estatística, ao nível de $5 \%$ de significância, pelo teste de Tukey.

TABELA 4 - Valores médios/desvio- padrão de Luminosidade (L*) das lichias 'Bengal' submetidas a tratamento térmico e sistemas de embalagens e armazenadas a $4 \mathrm{oC}$ por 21 dias.

\begin{tabular}{cccccccc}
\hline \multirow{2}{*}{ Trat. } & \multicolumn{7}{c}{ Tempo de estocagem (dias) } \\
\cline { 2 - 8 } & 2 & 5 & 8 & 12 & 15 & 18 & 21 \\
\hline T1 & $41,4 / 1,3 \mathrm{ABC}$ & $41,1 / 1,2 \mathrm{~A}$ & $40,4 / 1,2 \mathrm{~A}$ & $43,1 / 2,9 \mathrm{~A}$ & $40,3 / 1,6 \mathrm{AB}$ & $42,1 / 1,7 \mathrm{AB}$ & $43,2 / 1,4 \mathrm{~A}$ \\
$\mathrm{~T} 2$ & $40,4 / 1,2 \mathrm{BC}$ & $41,8 / 1,7 \mathrm{~A}$ & $41,4 / 2,1 \mathrm{~A}$ & $42,9 / 3,0 \mathrm{~A}$ & $39,6 / 2,3 \mathrm{AB}$ & $42,0 / 1,1 \mathrm{AB}$ & $42,8 / 1,6 \mathrm{~A}$ \\
$\mathrm{~T} 3$ & $40,3 / 0,5 \mathrm{C}$ & $41,2 / 1,7 \mathrm{~A}$ & $40,2 / 2,1 \mathrm{~A}$ & $40,8 / 1,4 \mathrm{~A}$ & $40,0 / 2,0 \mathrm{AB}$ & $40,9 / 1,5 \mathrm{AB}$ & $41,7 / 0,9 \mathrm{~A}$ \\
$\mathrm{~T} 4$ & $41,8 / 1,3 \mathrm{ABC}$ & $42,0 / 1,3 \mathrm{~A}$ & $40,7 / 2,0 \mathrm{~A}$ & $43,2 / 3,1 \mathrm{~A}$ & $37,9 / 1,8 \mathrm{~B}$ & $42,0 / 2,3 \mathrm{AB}$ & $44,6 / 1,6 \mathrm{~A}$ \\
$\mathrm{~T} 5$ & $42,9 / 1,6 \mathrm{AB}$ & $43,1 / 2,0 \mathrm{~A}$ & $40,3 / 1,7 \mathrm{~A}$ & $43,1 / 1,5 \mathrm{~A}$ & $39,7 / 1,7 \mathrm{AB}$ & $44,1 / 1,7 \mathrm{BB}$ & $43,5 / 3,5 \mathrm{~A}$ \\
$\mathrm{~T} 6$ & $42,5 / 1,7 \mathrm{ABC}$ & $42,1 / 1,7 \mathrm{~A}$ & $41,0 / 1,2 \mathrm{~A}$ & $42,0 / 1,1 \mathrm{~A}$ & $39,5 / 1,0 \mathrm{AB}$ & $42,8 / 1,8 \mathrm{AB}$ & $43,5 / 3,5 \mathrm{~A}$ \\
$\mathrm{~T} 7$ & $42,9 / 2,0 \mathrm{AB}$ & $40,6 / 1,4 \mathrm{~A}$ & $41,1 / 1,0 \mathrm{~A}$ & $43,9 / 1,0 \mathrm{~A}$ & $40,3 / 1,4 \mathrm{AB}$ & $42,7 / 1,7 \mathrm{AB}$ & $44,5 / 2,0 \mathrm{~A}$ \\
T8 & $43,4 / 1,2 \mathrm{~A}$ & $43,2 / 2,6 \mathrm{~A}$ & $43,2 / 1,8 \mathrm{~A}$ & $42,9 / 1,1 \mathrm{~A}$ & $41,0 / 0,8 \mathrm{~A}$ & $43,3 / 0,7 \mathrm{~A}$ & $45,0 / 2,4 \mathrm{~A}$ \\
\hline
\end{tabular}

Medias/desvio-padrão seguidas da mesma letra na vertical não apresentam diferença estatística, ao nível de $5 \%$ de significância, pelo teste de Tukey. 


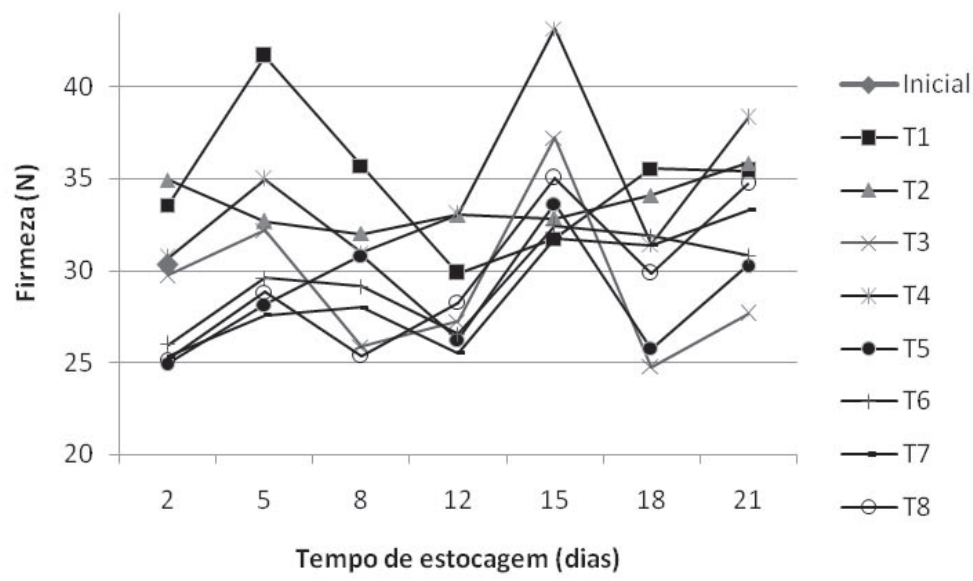

FIGURA 1- Valores médios de firmeza das lichias 'Bengal' ao longo do tempo de estocagem.

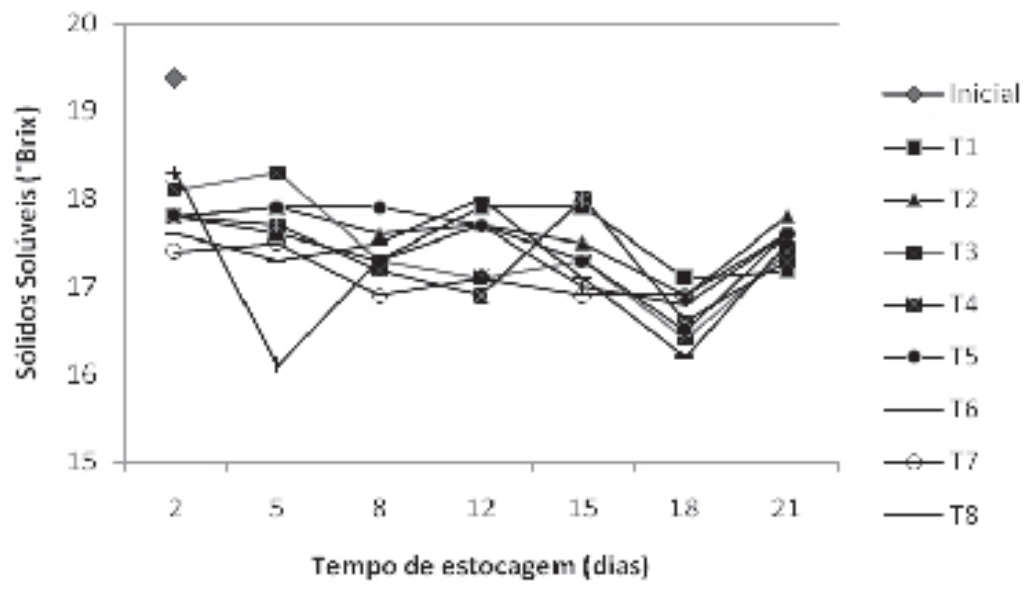

FIGURA 2- Valores médios de sólidos solúveis das lichias ‘Bengal' ao longo da estocagem.

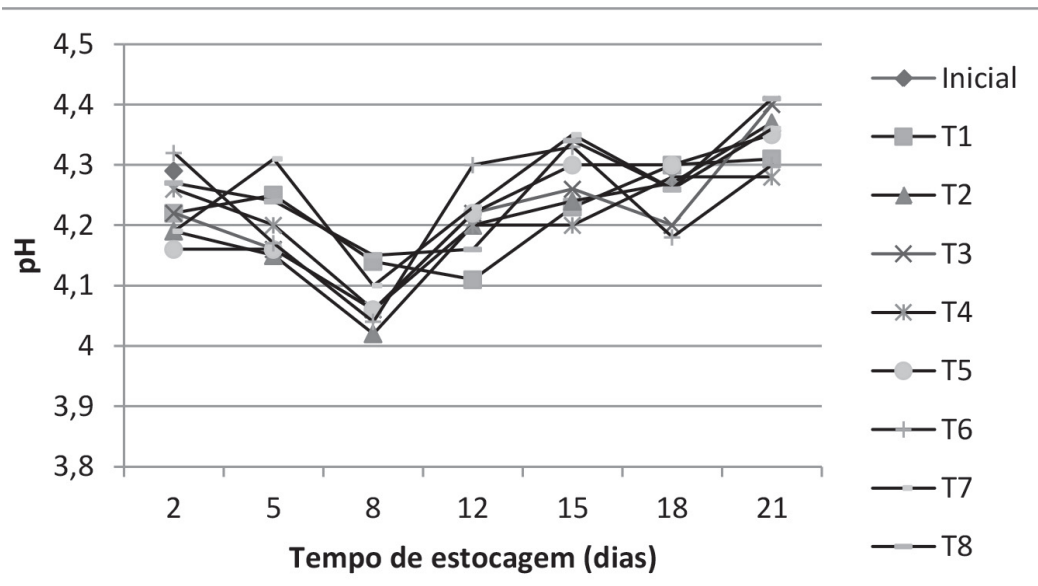

FIGURA 3-Valores médios de $\mathrm{pH}$ das lichias 'Bengal' ao longo da estocagem. 


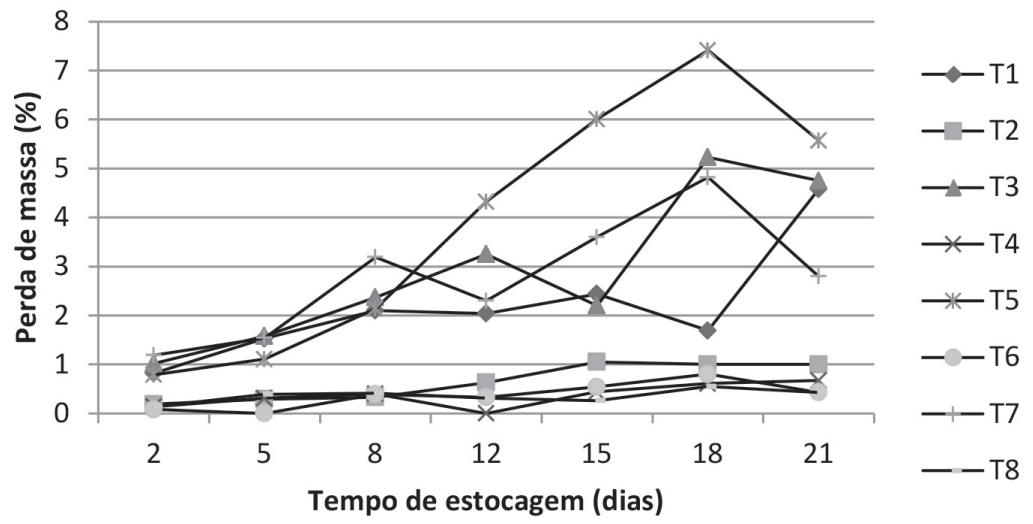

FIGURA 4- Valores médios de perda de massa em lichias 'Bengal' ao longo do estudo.

\section{CONCLUSÃO}

Observa-se eficiência dos tratamentos utilizados nas lichias comprovada pela estabilidade física e química observada durante 21 , dias estocadas a $4^{\circ} \mathrm{C}$.

A avaliação visual mostra que os tratamentos T5-BT, T6-BF, T7-BT e T8-BF são inaceitáveis a partir de 15 dias, e os tratamentos T1- BT, T2-BF, T3-BT e T4-BF, aceitáveis até o final do estudo, mantidos a $4^{\circ} \mathrm{C}$.

Os melhores tratamentos para a manutenção da cor vermelha da lichia a $4{ }^{\circ} \mathrm{C}$, ao final deste estudo, são os tratamentos T1- BT (sem tratamento hidrotérmico) e T4-BF (água a $50^{\circ} \mathrm{C}$ por $20 \mathrm{~s}$ ).

As amostras T2-BF (sem tratamento) e as tratadas com água a $55^{\circ} \mathrm{C}$ por $20 \mathrm{~s}$ ( T4-BF) apresentam os maiores valores de firmeza e as menores perdas de massa, demonstrando que o filme de PVC proporciona maior proteção quanto ao murchamento e à perda de umidade.

De acordo com este estudo, os tratamentos com ácido cítrico a $60 \%$ provocam manchas rosadas na superfície das lichias.

\section{AGRADECIMENTOS}

Ao Sr. Renato Mindlin Loeb, pela contratação do estudo, fornecimento de amostras e autorização para publicação dos dados, e a todos os da equipe do GEPC/ITAL.

\section{REFERÊNCIAS}

ALfEnAS, A.C.; MAFIA, R.G. Métodos em fitopatologia. Viçosa: Ed. UFV, 2007. 382p.

CARO, Y.; JOAS, J. Postharvest control of litchi pericarp browning (cv. Kwai Mi) by combined treatments of chitosan and organic acids II. Effect of the initial water content of pericarp. Postharvest Biology and Technology, Amsterdam, v.38, n.2, p. 137-144, 2005.

CAVALLARI, L. L. Florescimento e frutificação em lichieiras. 2009. 43 f. Dissertação (Mestre em Agronomia) - Faculdade de Ciências Agrárias e Veterinária, Universidade Estadual Paulista, Jaboticabal, 2009.

CEAGESP-HORTIBRASIL - Centro de Qualidade em Horticultura. In: BODNAR, L.; ROCHA, I.L. Evolução da comercialização da lichia na CEAGESP. Disponível em: $\leq$ www.hortibrasil.org.br>. Acesso em: 25 fev. 2014.

CHItARRA, M. I. F.; CHITARRA, A. B. Póscolheita de frutas e hortaliças: fisiologia e manuseio. 2.ed. Lavras: UFLA, 2005. 785p.

DUCAMP-COLLIN, M.N.; RAMARSON, H.; LEBRUN, M.; SELF, G.; REYNES, M. Effect of citric and chitosan on maintaining red colouration of litchi fruit pericarp. Postharvest Biology and Technology, Amsterdam, v.49, n.2, p. 241-246, 2008.

EMBRAPA. Rede regional de agroecologia. Lichia. Jaguariúna: Embrapa Meio ambiente, 2009.3p. 
FERREIRA, V. L. P. Colorimetria em alimentos. Campinas: ITAL, 1991. 43p.

HOLCROFT, D. M.; MITCHAM, E. J. Postharvest physiology and handling of litchi (Lichi chinensis Sonn.). Postharvest Biology and Technology, Amsterdam, v.9, p. 265-281, jun. 1996.

HUANG, X.M.; WANG, H.C.; YUAN, W.Q.; LU, J.M. A study of rapid senescence of detached litchi: roles of water loss and calcium. Postharvest Biology and Technology, Amsterdam, v.36, n.2, p.177-189, 2005.

INSTITUTO ADOLFO LUTZ. Normas analíticas do Instituto Adolfo Lutz: métodos químicos e físicos para análise de alimentos. 4. ed. São Paulo, 2008. v.1, 20p. (Versão eletrônica).

JIANG, Y.; DUAN.; XUEWU, D.; JOYCE, D.; ZHANG, Z.; LI, J. Advances in understanding of enzymatic browning in harvested lichi fruit. Food Chemistry, London, v.88, n.3, p.443-446, 2004.

JIANG, Y.M.; CHEN, F. A study on polyamiane change and browning of fruit during cold storage of litchi (Litchi chinensis Sonn.). Postharvest Biology and Technology, Amsterdam, v. 5, n.3, p. 245-250, 1995. corrigir p.3 linha 20 de JIANG; CHEN, 1994 para JIANG; CHEN, 1995

JOAS, J.; CARO, Y.; DUCAMP, M. N.; REYNES, M. Postharvest control of pericarp browning of litchi fruit (Litchi chinensis Sonn cv Kwai Mi) by treatment with chitosan and organic acids I. Effect of $\mathrm{pH}$ and pericarp dehydration. Postharvest Biology and Technology, Amsterdam, v. 38, n.2, p.128-136, 2005.

LICHTER, A.; DVIR, O.; ROT, I.; AKERMAN, M.; REGEV, R.; WIESBLUM, A.; FALLIK, E.; ZAUBERMAN, G.; FUCHS, Y. Hot water brushing: an alternative method to $\mathrm{SO}_{2}$ fumigation for color retention of lichi fruits. Postharvest Biology and Technology, Amsterdam, v.18, n.3, p.235-244, 2000.

MENEZES, M. Guia prático para fungos fitopatogênicos, 2.ed. rev. e ampl. Recife: UFRPE, Imprensa Universitária, 2004. 183p.
MOTTA, E. L. Avaliação da composição nutricional e atividade antioxidante de Litchi Chinensis (Lichia) cultivada no Brasil. 2009. 100 f. Dissertação (Mestrado) - Faculdade de Farmácia da Universidade Federal do Rio de Janeiro, Rio de Janeiro, 2009

SILVA, D. F. P.; LINS, L. C. R.; CABRINI, E. C.; BRASileiro, B. G.; SAlOMÃO, L. C. C. Influence of the use of acids and films in post-harvest lychee conservation. Revista Ceres, Viçosa, MG, v.59, n.6, p.859-866, 2012.

SIVAKUMAR, D.; KORSTEN, L. Influence of modified atmosphere packaging and postharvest treatments on quality retention of litchi cv. Mauritius. Postharvest Biology and Technology, Amsterdam, v.41, n.2, p.135-142, 2006.

TODA FRUTA. Pós-colheita de lichia. Disponível em: <http://www. todafruta.com.br/todafruta $>$. Acesso em: 10 maio 2013.

ZHANG, D.; QUANTICK, C. P. Effects of chitosan coating on enzymatic browning and decay during postharvest storage of lichi (Litchi chinensis Sonn.) fruit. Postharvest Biology and Technology, Amsterdam, v.12, n.2, p.195-202, 1997.

ZHANG, D.; QUANTICK, P. C.; GRIGOR, J. M. Changes in phenolic compounds in Litchi (Litchi chinensis Sonn.) fruit during postharvest storage. Postharvest Biology and Technology, Amsterdam, v.19, n.2, p.165-172, 2000.

ZHENG, X.; TIAN, S. Effect of oxalic acido on control of postharvest brownin $\mathrm{g}$ of litchi fruit. Food Chemistry, London, v.96, n.4, p.519-523, 2006. 